Supplement of Hydrol. Earth Syst. Sci., 25, 1117-1131, 2021

https://doi.org/10.5194/hess-25-1117-2021-supplement

(c) Author(s) 2021. This work is distributed under

the Creative Commons Attribution 4.0 License.

(c) (1)

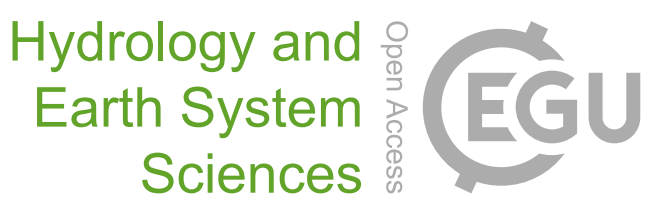

Supplement of

\title{
Soil dielectric characterization during freeze-thaw transitions using L-band coaxial and soil moisture probes
}

\author{
Alex Mavrovic et al. \\ Correspondence to: Alex Mavrovic (alex.mavrovic@uqtr.ca)
}

The copyright of individual parts of the supplement might differ from the CC BY 4.0 License. 
Table S1: Temperature offsets of the thawing and freezing transitions for the real permittivity. The soil temperature offsets were evaluated at maximum transition rate. The average temperature offset is given along with the range of values. $\Delta T_{\text {offset }}=T_{\text {offset }}(H P)-T_{\text {offset }}($ OECP $)$.

\begin{tabular}{|c|c|c|c|c|c|c|}
\hline \multirow{2}{*}{ Soil type } & \multicolumn{3}{|c|}{ Thawing } & \multicolumn{3}{|c|}{ Freezing } \\
\hline & $\begin{array}{l}\text { OECP } \\
{ }^{\circ} \mathrm{C}\end{array}$ & $\begin{array}{l}\mathrm{HP} \\
{ }^{\circ} \mathrm{C}\end{array}$ & $\begin{array}{c}\Delta \mathrm{T}_{\text {offset }} \\
{ }^{\circ} \mathrm{C}\end{array}$ & $\begin{array}{l}\mathrm{OECP} \\
{ }^{\circ} \mathrm{C}\end{array}$ & $\begin{array}{l}\mathrm{HP} \\
{ }^{\circ} \mathrm{C}\end{array}$ & $\begin{array}{c}\Delta \mathrm{T}_{\text {offset }} \\
{ }^{\circ} \mathrm{C}\end{array}$ \\
\hline Organic & $\begin{array}{c}3.43 \\
{[3.4-3.5]}\end{array}$ & $\begin{array}{c}3.82 \\
{[3.1-4.4]}\end{array}$ & +0.39 & $\begin{array}{c}-2.5 \\
-[2.6-2.4]\end{array}$ & $\begin{array}{c}-2.66 \\
-[3.6-2.0]\end{array}$ & -0.16 \\
\hline Sandy Loam & $\begin{array}{c}2.05 \\
{[2-2.1]}\end{array}$ & $\begin{array}{c}2.65 \\
{[2.5-2.8]}\end{array}$ & +0.60 & $\begin{array}{c}-2.75 \\
-[2.8-2.7]\end{array}$ & $\begin{array}{c}-2.83 \\
-[2.9-2.8]\end{array}$ & -0.08 \\
\hline Loamy Sand & $\begin{array}{c}2.00 \\
{[1.9-2.2]}\end{array}$ & $\begin{array}{c}2.10 \\
{[2.1-2.1]}\end{array}$ & +0.10 & $\begin{array}{c}-1.80 \\
-[2.1-1.5]\end{array}$ & $\begin{array}{c}-2.20 \\
-[2.3-2.1]\end{array}$ & -0.30 \\
\hline Clay Loam & $\begin{array}{c}2.90 \\
{[2.8-3.0]}\end{array}$ & $\begin{array}{c}3.10 \\
{[3.0-3.2]}\end{array}$ & +0.20 & $\begin{array}{c}-1.50 \\
-[1.5-1.5]\end{array}$ & $\begin{array}{c}-1.77 \\
-[1.8-1.7]\end{array}$ & -0.27 \\
\hline
\end{tabular}

Table S2: Same as Table S1 but for imaginary permittivity

\begin{tabular}{|c|c|c|c|c|c|c|}
\hline \multirow[b]{2}{*}{ Soil type } & \multicolumn{3}{|c|}{ Thawing } & \multicolumn{3}{|c|}{ Freezing } \\
\hline & $\begin{array}{l}\mathrm{OECP} \\
{ }^{\circ} \mathrm{C}\end{array}$ & $\begin{array}{l}\mathrm{HP} \\
{ }^{\circ} \mathrm{C}\end{array}$ & $\begin{array}{l}\Delta \mathrm{T}_{\text {offset }} \\
{ }^{\circ} \mathrm{C}\end{array}$ & $\begin{array}{l}\text { OECP } \\
{ }^{\circ} \mathrm{C}\end{array}$ & $\begin{array}{l}\mathrm{HP} \\
{ }^{\circ} \mathrm{C}\end{array}$ & $\begin{array}{l}\Delta \mathrm{T}_{\text {offset }} \\
{ }^{\circ} \mathrm{C}\end{array}$ \\
\hline Organic & $\begin{array}{c}3.43 \\
{[3.4-3.5]}\end{array}$ & $\begin{array}{c}4.26 \\
{[3.7-4.8]}\end{array}$ & +0.83 & $\begin{array}{c}-2.50 \\
-[2.6-2.4]\end{array}$ & $\begin{array}{c}-2.70 \\
-[3.6-2.0]\end{array}$ & -0.20 \\
\hline Sandy Loam & $\begin{array}{c}2.00 \\
{[2.0-2.0]}\end{array}$ & $\begin{array}{c}2.75 \\
{[2.7-2.8]}\end{array}$ & +0.75 & $\begin{array}{c}-2.75 \\
-[2.8-2.7]\end{array}$ & $\begin{array}{c}-2.75 \\
-[2.8-2.7]\end{array}$ & 0 \\
\hline Loamy Sand & $\begin{array}{c}2.00 \\
{[1.9-2.2]}\end{array}$ & $\begin{array}{c}2.10 \\
{[2.1-2.1]}\end{array}$ & +0.10 & $\begin{array}{c}-1.80 \\
-[2.1-1.5]\end{array}$ & $\begin{array}{c}-2.80 \\
-[2.6-2.3]\end{array}$ & -1.00 \\
\hline Clay Loam & $\begin{array}{c}2.90 \\
{[2.8-3.0]}\end{array}$ & $\begin{array}{c}3.17 \\
{[3.1-3.2]}\end{array}$ & +0.27 & $\begin{array}{c}-1.50 \\
-[1.5-1.5]\end{array}$ & $\begin{array}{c}-1.43 \\
-[1.5-1.3]\end{array}$ & +0.07 \\
\hline
\end{tabular}

